\title{
Cooperative Scheduling, Precoding, and Optimized Power Allocation for LTE-Advanced CoMP Systems
}

\author{
Rana A. Abdelaal, Khaled Elsayed, and Mahmoud H. Ismail \\ Department of Electronics and Communications Engineering, Faculty of Engineering, \\ Cairo University, Giza 12613, Egypt \\ \{rabdelaal, Khaled, mhismail\}@iieee.org
}

\begin{abstract}
Recent research has shown that coordinated multipoint (CoMP) transmission can provide significant gains in terms of overall throughput of cellular systems. The main purpose of this paper is to enhance the overall cell throughput and to optimize the power consumption in LTE-Advanced (LTE-A) systems using CoMP. In particular, we present scheduling, precoding and power allocation (PA) algorithms based on the signal-to-leakage-plus-noise-ratio (SLNR) for the CoMP downlink. The proposed scheduling and precoding algorithm selects the user equipment (UEs) that can efficiently share the same resource block (RB) without degrading the overall throughput by using the SLNR metric. Additionally, we propose PA algorithms that significantly improve the overall throughput and reduce the power consumption. The $P A$ algorithms are based on solving a set of constrained convex optimization problems using the log-barrier function penalty method based on using the Newton method. We evaluate the proposed PA algorithms by comparing them to equal power allocation (EPA). Performance evaluation results show that the proposed SLNR-based PA algorithms provide considerable performance gains in terms of the overall system throughput and also are shown to have even less power consumption compared to the typical EPA.
\end{abstract}

Keywords- Power Allocation; Scheduling; Precoding; Coordinated Multi- point; LTE; Interference Mitigation, Newton's Method.

\section{INTRODUCTION}

The capacity of modern wireless cellular networks is mainly limited by interference. In cellular systems, a geographical region is typically divided into cells, which handle interference through the use of pre-defined frequency reuse patterns [1] and [2]. Moreover, nowadays, cellular networks demand power consumption reduction with the aim of improving the energy efficiency. Careful power allocation (PA) plays an important role in wireless networks. This can be demonstrated by controlling the transmitted power intended for each user equipment (UE) in the cellular system, which not only helps in reducing the overall power consumed, but also in enhancing the overall throughput. This is due to the fact that minimizing the transmission power for a specific UE can lead to reducing the interference to other UEs and thus, increases the achievable throughput.

One of the promising techniques in Long Term EvolutionAdvanced (LTE-A) is coordinated multi-point (CoMP) transmission, which is introduced in an attempt to meet the high data rate requirements of IMT-Advanced [3]. The basic idea of CoMP is to mitigate interference through cooperation between several remote radio equipments (RREs), which can be connected to a central BS or eNB. Since the interface connecting the central BS and the RREs can be implemented through the use of optical fibers or via dedicated radio, highspeed transfer of signals is possible. This cooperation results in a distributed form of MIMO, thus enhancing spectral efficiency. In CoMP systems, two approaches are often considered. The first approach is coordinated scheduling (CS) where the data is transmitted from one RRE at a time with scheduling decisions being made with coordination between all RREs. The second approach is joint processing (JP) where the data is made available at each RRE and is transmitted from several RREs simultaneously to each UE [4].

Joint scheduling and PA schemes have been investigated in numerous previous works. For instance, in [5], a scheduling strategy has been proposed within the framework of cooperative cells. Equal power allocation (EPA) among the UEs in each cell has been assumed, which is not efficient since the UEs have different channels and they can better utilize the available energy through optimized PA. In [6] and [7], a PA algorithm has been proposed that improves the network throughput and power efficiency. However, it assumes that each UE is associated to the BS that has the smallest path loss, which does not guarantee the best scheduling of UEs because scheduling decisions should take both the signal and the interference into account. Also, the model assumes only a single $\mathrm{RB}$, which impacts the applicability of the algorithm in practical networks.

In this paper, we propose joint scheduling, precoding, and PA algorithms that can significantly improve the overall throughput as well as the energy efficiency. Our proposed algorithms are based on the signal-to-leakage-plus-noise ratio (SLNR), and are therefore inherently less complex than algorithms based on signal-to-interference-plus-noise ratio (SINR), which is very desirable in practical networks [8]. We tackle the PA problem from three different perspectives. The first one is what we call the Optimal Power Allocation (OPA), in which we solve a coupled problem with two power constraints at the same time. The first constraint is per RRE and the second one is per RB. The second proposed PA algorithm is called the Power Allocation per RRE (PAR), where the problem is solved for each RRE independently, which definitely reduces the complexity of the coupled problem. Finally, we propose an iterative solution for the PA problem that is solved for each RB independently and we call

This work is a part of the $4 \mathrm{G}++$ project supported by the National Telecom

Regularity Authority of Egypt. 
it the Iterative Power Allocation per RB (IPA). The three algorithms have a common ground, which is maximizing the overall throughput and also minimizing the total power consumption. It worth mentioning that, real-time implementation of the three proposed algorithms is possible since eNBs can be equipped with good processors. We also evaluate the three different algorithms for both CS-CoMP and JP-CoMP schemes. In this paper, we solve the proposed power allocation optimization problems via Newton's method with logarithmic barrier.

The remainder of the paper is organized as follows: In Section II, we describe the system model considered in the paper. Section III describes the scheduling and precoding algorithm. In Section IV, we explain the proposed power allocation algorithms. In Section V, we evaluate our proposed algorithms via simulations. Finally, we draw the main conclusions of the paper in Section VI.

\section{SYSTEM MODEL}

We consider a cellular system where each cell consists of one eNB, $M$ RREs under its control, and serves $K$ singleantenna UEs. There exists $N$ RBs in the system and each of them may be assigned to serve one or more UEs. The overall transmit power available for each RRE is equal to $P$. The proposed schemes exploit the SLNR metric for performing the scheduling, precoding, and PA. The SLNR $\left(\beta_{k}\right)$ at the $k$ th UE over the $n$th RB can be expressed as:

$$
\beta_{k}=\frac{P_{k, n}\left|\underline{\boldsymbol{h}}_{\boldsymbol{k}} \underline{\boldsymbol{w}}_{\boldsymbol{k}}\right|^{2}}{\sum_{k^{\prime} \neq k} P_{k, n}\left|\underline{\boldsymbol{h}}_{\boldsymbol{k}^{\prime}} \underline{\boldsymbol{w}}_{\boldsymbol{k}}\right|^{2}+\left|\eta_{k}\right|^{2}},
$$

where $P_{k, n}$ is the power allocated to the $k$ th UE over the $n$th $\mathrm{RB}, \underline{\boldsymbol{h}}_{\boldsymbol{k}}$ is the $1 \times M$ complex channel vector of the links between the $k$ th UE and all $M$ RREs of the CoMP cell, $\eta_{k}$ is the additive white Gaussian noise at the $k$ th $\mathrm{UE}$, and $\underline{\boldsymbol{w}}_{\boldsymbol{k}}$ is an $M \times 1$ weighting (precoding) vector that shapes the data transmitted from the $M$ RREs to the $k$ th UE. The numerator of (1) represents the signal intended for the $k$ th UE and the first term in the denominator represents the leakage on other UEs due to the signal intended for the $k$ th UE. As shown in (1), the SLNR of the $k$ th UE depends only on the weighting vector of this UE. On the other hand, the SINR of the $k$ th UE depends on the weighting vectors of all other UEs. Hence, using SLNR leads to a decoupled optimization problem and admits an analytical closed form solution unlike the case of using the SINR. The choice of the weighting vectors $\left\{\underline{\boldsymbol{w}}_{\boldsymbol{k}}, k=\right.$ $1,2, \ldots, K\}$ of the UEs will be targeting the maximization of the SLNR:

Maximize $\beta_{k}$ Subject to $\left\|\underline{\boldsymbol{w}}_{\boldsymbol{k}}\right\|^{2}=1$.

In case of the CS scheme, the weighting vector determines which RRE should serve a specific UE. Since in CS, each UE is served by only one RRE then all the elements in $\underline{\boldsymbol{w}}_{\boldsymbol{k}}$ are zeros except only one element will be equal to unity, which corresponds to the serving RRE. The index of the serving RRE can be easily obtained by solving the optimization problem in (2) through a simple exhaustive search procedure. On the other hand, in case of the JP scheme, the same data packet is sent to a specific UE from all RREs and thus $\underline{\boldsymbol{w}}_{\boldsymbol{k}}$ is not easily obtained as in the case of CS. This optimization problem has been solved in [9] and the solution was found to be:

$$
\underline{\boldsymbol{w}}_{\boldsymbol{k}}=\text { max eig. vec. }\left(\left(\left|\eta_{k}\right|^{2} \mathbf{I}_{M}+\widehat{\mathbf{H}}_{k}^{*} \widehat{\mathbf{H}}_{k}\right)^{-1} \underline{\boldsymbol{h}}_{k}^{*} \underline{\boldsymbol{h}}_{\boldsymbol{k}}\right) \text {, }
$$

where $\mathbf{I}_{\mathrm{M}}$ donates the $M \times M$ identity matrix, $\underline{\boldsymbol{w}}_{\boldsymbol{k}}$ is the eigenvector corresponding to the maximum eigenvalue of the matrix computed in (3), and $\widehat{\mathbf{H}}_{k}$ is a $(K-1) \times M$ matrix given by:

$$
\widehat{\mathbf{H}}_{k}=\left[\begin{array}{llll}
\underline{\boldsymbol{h}}_{1} & \underline{\boldsymbol{h}}_{2} & \cdots & \underline{\boldsymbol{h}}_{\boldsymbol{k}-1} \underline{\boldsymbol{h}}_{\boldsymbol{k}+\mathbf{1}} \cdots \underline{\boldsymbol{h}}_{\boldsymbol{K}}
\end{array}\right]^{T} .
$$

Having selected the appropriate precoding vectors, for the $n$th $\mathrm{RB}$, a set $\delta_{n} \subset\{1,2, \ldots, K\}$ of UEs will be constructed to share this RB. The task of the scheduling stage is now to select the UEs that can efficiently share the same RB without degrading the overall throughput. In that way, the overall throughput will be enhanced and the available bandwidth will be efficiently utilized.

\section{SCHEdUling AND Precoding ALgORITHM}

We now explain the scheduling and precoding algorithm with the objective of maximizing the overall throughput and maintaining fairness among UEs. It is very important to note here that since our scheduling and precoding algorithm uses the SLNR metric, which uses the same power in the numerator and denominator as shown in (1), the effect of PA in the scheduling and precoding phase will not be significant since noise power is typically small compared with leakage value. Thus, it is anticipated that decoupling the scheduling/precoding and the PA problem for the UEs belonging to the same set $\delta_{n}$ will provide a good basis for simplified approach without sacrificing accuracy.

For the $n$th RB, the set of UEs $\mathcal{S}_{\mathrm{n}}$ is initialized to the empty set. The first step in the algorithm is that the UE with maximum SLNR will be chosen and set to be the first element in the set $\mathcal{S}_{\mathrm{n}}$. Then, the leakage value vector from set $\left(\mathcal{S}_{\mathrm{n}}\right)$ in the direction of the rest of UEs is computed. This vector represents the amount of leakage from the set $\left(\mathcal{S}_{\mathrm{n}}\right)$ to the rest of UEs. Leakage refers to the interference caused by the signals intended for the UEs belonging to the set $\left(\mathcal{S}_{\mathrm{n}}\right)$ on the remaining UEs, i.e., leakage is a measure of how much signal power leaks into the other UEs. We previously discussed the leakage concept in [8]. Then the UE with the least amount of leakage will be added to the set $\mathcal{S}_{\mathrm{n}}$. In that step, the UE that will be affected the least will share the RB with the UEs belonging to the set. This UE will be selected according to [8]. Finally, the algorithm will continue adding UEs to $\mathcal{S}_{\mathrm{n}}$ till a certain condition is satisfied (a certain threshold is reached or a certain marginal utility function with/without look ahead does not increase) as we proposed earlier in [8].

\section{Power Allocation Algorithms}

In this section, we investigate three PA algorithms for both CoMP schemes, aiming at minimizing the overall power consumption of the entire network while maximizing the overall data rate. As mentioned earlier, each RRE has a total power constraint $(P)$ and serves several UEs and each RRE will initially divide its total power over its scheduled UEs. 


\section{A. Optimal Power Allocation (OPA) Algorithm}

The OPA algorithm is designed to deliver high SLNR values for all UEs. This SLNR balancing along with applying two constraints (per RRE and per RB power constraints) ensures achieving high throughput gains and reducing the total power consumption at the same time. The OPA algorithm seems to be very complicated due to its coupled nature, however, it can be considered as a benchmark for evaluation to which other algorithms could be compared. It can also be practically applied in small-scale wireless networks where the numbers of available RBs and UEs are considerably small. In contrast, in Sections IV.B and IV.C, we propose two algorithms to solve the PA problem in large-scale networks.

We mentioned earlier that each RRE has a total power $(P)$ to be divided among its scheduled UEs, so each UE has been already allocated a portion of its own serving RRE total power. We consider the summation of the powers of the UEs belonging to the $n$th set (the $n$th set being the set of UE's sever by RRE $n$ ) as the power constraint per the $n$th RB for the power allocation problem at hand. For example, if we have three RREs, the first is serving three UEs, the second is serving two UEs, and the third is serving one UE and assuming that the $n$th RB is shared among three UEs (one UE served by each RRE, assuming CS and equal power allocation, for example), the first UE (served by the first RRE) will be allocated $P / 3$, the second UE will be allocated $P / 2$, and the third one will be allocated $P$. We can consider that $P / 3+P / 2+P$ as the power constraint for the $n$th RB. We can call it the maximum power per RB $\left(\rho_{n}\right)$ since the UEs are sharing the same RB. Now, in the above example, we have $1.83 P$ as our per $\mathrm{RB}$ constraint. The proposed power constraint per RB is, in fact, an artificially constructed constraint. Note that, in this example, CS has been assumed, but JP can be applied as well. The allocated power per RB for a single RRE has been considered before in [10] and [11] where it was required to divide the total power of an RRE over its available RBs. However, the allocated power per $\mathrm{RB}$ in case of multiple RREs has not been used in previous works. The OPA problem can now be formulated as follows:

$$
\begin{gathered}
\max _{P_{k, n}} f=\sum_{n=1}^{N} \sum_{k \in \mathcal{S}_{n}} \frac{P_{k, n}\left|\underline{h}_{k} \underline{w}_{k}\right|^{2}}{\sum_{k^{\prime} \in \mathcal{S}_{n}, k^{\prime} \neq k} P_{k, n}\left|\underline{h}_{k^{\prime}} \underline{w}_{k}\right|^{2}+\eta_{k}^{2}}, \\
\text { subject to } \sum_{k \in \mathcal{S}_{n}} P_{k, n} \leq \rho_{n}=\sum_{m \in \mathcal{T}_{n}} P /\left|\mathcal{K}_{m}\right| \forall n \in\{1,2, \ldots, N\}, \\
\text { and } \sum_{k \in \mathcal{K}_{m}} \sum_{n \in \mathcal{N}_{k, m}} P_{k, n} \leq P \forall m \in\{1,2, \ldots, M\},
\end{gathered}
$$

where $\mathcal{T}_{n}$ is the set of RREs serving the UEs belonging to $\mathcal{S}_{n}$, $\rho_{n}$ is the total power per the $n$th RB, $P$ is the total power per the $m$ th RRE, $\mathcal{N}_{k, m}$ is the set of RBs allocated to the $k$ th UE and served by the $m$ th RRE, $\mathcal{K}_{m}$ is the set of UEs served by the $m$ th RRE and $\left|\mathcal{K}_{m}\right|$ is the cardinality of the set $\mathcal{K}_{m}$ (the number of scheduled UEs of the $m$ th RRE), and $\sum_{m=1}^{M}\left|\mathcal{K}_{m}\right| \leq K$.

The optimization problem (5) is concave since the second derivative of the objective function is non-negative and the constraints are linear. The solution to the optimization problem defined by (5) (as well the other problems that will be defined in the sequel) can be found using Newton with logarithmic barrier penalty method, which is one of the interior point methods used for solving convex optimization problems with inequality constraints [12].

The outline of the Newton with logarithmic barrier method is as follows:

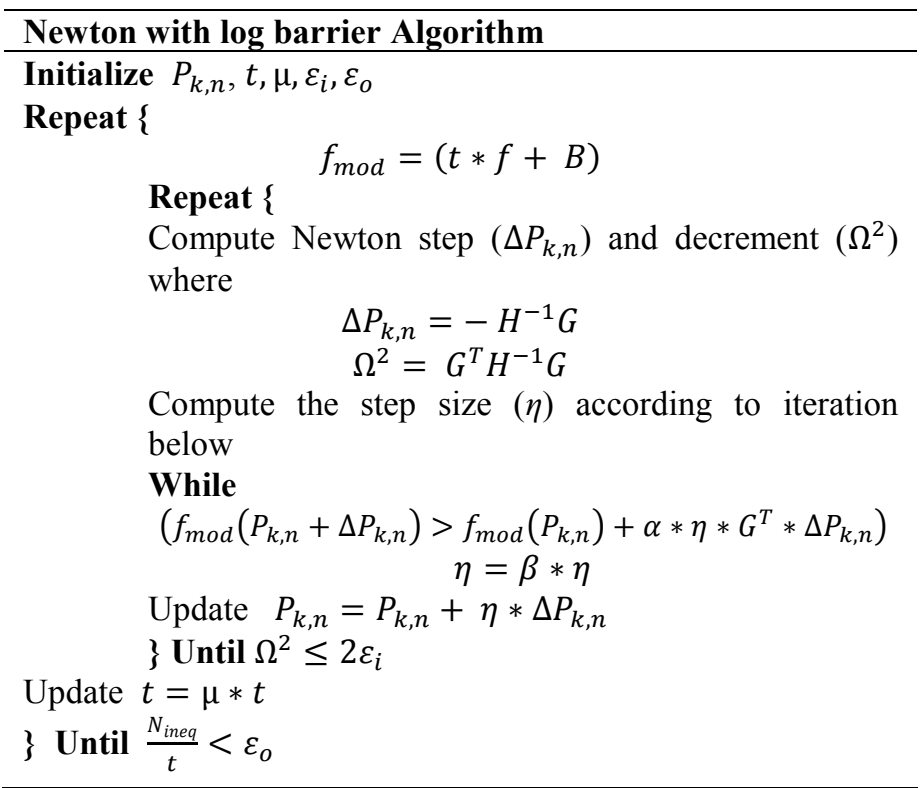

In the above algorithm, $B$ is the barrier function to be added to the objective function $f$ to formulate the modified objective function $f_{\text {mod }}, N_{\text {ineq }}$ is the number of the inequality constraints, $\varepsilon_{o}$ is the outer desired accuracy (i.e., accepted tolerance), $\varepsilon_{i}$ is the inner accuracy. Note that, the desired accuracy of the inner loop and the outer loop can be different. Also, $G$ is the gradient of the modified function, $H$ is the Hessian of the modified function, and $t$ is a parameter that controls the number of iterations for achieving the desired accuracy. It is worth noting here, that $t$ is not a fixed parameter, it is a variable parameter that depends on the iteration number. The barrier function can be found as:

$$
\begin{gathered}
B_{O P A}\left(P_{k, n}\right)=\sum_{n=1}^{N}\left\{-\log \left(\rho_{n}-\sum_{k \in S_{n}} P_{k, n}\right)\right\}+\sum_{m=1}^{M}\{-\log (P- \\
\left.\left.\sum_{k \in \mathcal{K}_{m}} \sum_{n \in \mathcal{N}_{k, m}} P_{k, n}\right)\right\}
\end{gathered}
$$

It is worth mentioning that the step size $(\eta)$ is computed via the backtracking line search algorithm [12], where $\beta$ a positive constant less than one and $\alpha$ is a positive constant less than 0.5 .

Now, the modified optimization problem will be:

$$
\max _{P_{k, n}} f_{O P A}
$$

where

$$
\begin{aligned}
f_{O P A}=t * \sum_{n=1}^{N} \sum_{k \in S_{n}} \frac{P_{k, n}\left|\underline{\boldsymbol{h}}_{k} \underline{\boldsymbol{w}}_{\boldsymbol{k}}\right|^{2}}{\sum_{k^{\prime} \in \mathcal{S}_{n}, k^{\prime} \neq k} P_{k, n}\left|\underline{\boldsymbol{h}}_{\boldsymbol{k}^{\prime}} \underline{\boldsymbol{w}}_{\boldsymbol{k}}\right|^{2}+\eta_{k}{ }^{2}} \\
+B_{O P A}\left(P_{k, n}\right)
\end{aligned}
$$

And by computing the gradient and the Hessian of (8), we can apply the Newton with logarithmic barrier method to get a solution to the optimization problem in (7). 
It is worth noting that, the OPA algorithm with the constraints on both the total power per RRE and the total power per RB turns the optimization problem into a highly coupled one, which is very complicated and intractable for a large system with a large number of UEs.

We now propose another two relaxed optimization problems; the first one can be solved for each RRE independently and the second one can be solved for each RB independently. We will use the OPA algorithm as a benchmark for evaluating the performance of the other two PA algorithms.

\section{B. Power Allocation Per RRE (PAR) Algorithm}

Instead of the approach followed by the OPA algorithm which is based on coupling the multicell PA for all UEs served by all RREs over all RBs at the same time, one may naturally conjecture that solving $M$ independent (one per RRE) PA optimization problems can provide good system performance while reducing the computational complexity. When doing so, the power allocation per RRE (PAR) algorithm can be considered as a sub-optimal but practical algorithm. It attempts to find the power allocated to each served UE subject to a constraint on the RRE's total power. With this assumption, the optimization problem in (5) will be decoupled and is concave since the power constraint is linear and the second derivative of the objective function can be shown to be non-negative. The PAR problem can thus be formulated as:

$$
\begin{gathered}
\max _{P_{k, n}} \sum_{k \in \mathcal{K}_{m}} \sum_{n \in \mathcal{N}_{k, m}} \frac{P_{k, n}\left|\underline{h}_{k} \underline{w}_{k}\right|^{2}}{\sum_{k^{\prime} \in \mathcal{S}_{n}, k^{\prime} \neq k} P_{k, n}\left|\underline{h}_{k} \underline{w}_{k}\right|^{2}+\eta_{k}{ }^{2}} \\
\text { subject to } \sum_{k \in \mathcal{K}_{m}} \sum_{n \in \mathcal{N}_{k, m}} P_{k, n} \leq P \forall m \in\{1,2, \ldots, M\}
\end{gathered}
$$

The PAR problem is solved for each RRE independently. The solution to this problem can also be found using Newton with log barrier penalty method as detailed before. We first define the barrier function to be added to the objective function as:

$$
B_{P A R}\left(P_{k, n}\right)=\sum_{m=1}^{M}\left\{-\log \left(P-\sum_{k \in \mathcal{K}_{m}} \sum_{n \in \mathcal{N}_{k, m}} P_{k, n}\right)\right\}
$$

Now, the modified optimization problem will be:

$$
\max _{P_{k, n}} f_{P A R}
$$

where

$$
\begin{aligned}
& f_{P A R}=\left(t * \sum_{k \in \mathcal{K}_{m}} \sum_{n \in \mathcal{N}_{k, m}} \frac{P_{k, n}\left|\underline{h}_{k} \underline{w}_{k}\right|^{2}}{\sum_{k^{\prime} \in S_{n}, k^{\prime} \neq k} P_{k, n}\left|\underline{h}_{k^{\prime}} \underline{w}_{k}\right|^{2}+\eta_{k}^{2}}\right. \\
& \left.+B_{P A R}\left(P_{k, n}\right)\right)
\end{aligned}
$$

And, by computing the gradient and the Hessian of (12), we can apply the Newton with logarithmic barrier method to find a solution to the optimization problem in (11).

It is assumed that each RRE will have the scheduling decisions of other RREs by means of coordination (i.e., it will be known to each RRE the sets to which its UEs belong). Although the PAR algorithm is applied to each RRE independently, it still depends on coordination between RREs. This is because the SLNR metric couples both the intended signal and the effect on other users in one expression. Hence, if each RRE considers maximizing the SLNR for only its served UEs, good SINR for all users should be attainable since other RREs will do exactly the same thing. It is worth noting that the PAR algorithm controls the signal intended to each UE, but it does not control the interference signal that is resulting from other UEs sharing the same RB. So, we need to investigate another power allocation algorithm that can control the powers of the UEs sharing the same RB in order to keep the ratio between useful power and undesired interference below a certain level for all UEs sharing the same RB.

\section{Iterative Power Allocation Per RB (IPA)}

In the iterative power allocation per RB (IPA) algorithm, we consider the UEs belonging to the same set (sharing the same RB) as the UEs over which the power should be divided. The reasoning behind this is that the power allocation of each UE within the set affects the whole set in terms of the throughput (because of the interference caused by any member of the set over the others).

Now, if we have $N$ RBs, we will need to solve the proposed optimization problem $N$ times independently. To do so, we propose removing the constraint that couples the RBs together (Per-RRE power constraint) in (5). Removing this constraint ensures transforming the highly coupled optimization problem in (5) into $N$ decoupled optimization problems. We will show later in the sequel how the per-RRE constraint can be taken into consideration. To wrap up, the IPA problem has two constraints but we will divide it into two small problems each one of them takes into consideration only one constraint at a time and then we will iterate over them until we reach a solution. This separation of the constraints with iteration over them can be considered as a simplified form of the main problem. The optimization problem can now be formulated as:

$$
\begin{aligned}
& \max _{P_{k, n}, k \in \mathcal{S}_{n}} \sum_{k \in \mathcal{S}_{n}} \frac{P_{k, n}\left|\underline{h}_{k} \underline{w}_{k}\right|^{2}}{\sum_{k^{\prime} \in \mathcal{S}_{n}, k^{\prime} \neq k} P_{k, n}\left|\underline{h}_{k^{\prime}} \underline{w}_{k}\right|^{2}+\eta_{k}{ }^{2}}, \\
& \text { subject to } \sum_{k \in \mathcal{S}_{n}} P_{k, n} \leq \rho_{n} \forall n \in\{1,2, \ldots, N\}
\end{aligned}
$$

The solution to our problem can now again be found using Newton with log barrier method. We define a barrier function to be added to the objective function as:

$$
B_{I P A}\left(P_{k, n}\right)=\sum_{n=1}^{N}\left\{-\log \left(\rho_{n}-\sum_{k \in S_{n}} P_{k, n}\right)\right\}
$$

Now, the modified optimization problem will be:

$$
\max _{P_{k, n}} f_{I P A}
$$

where

$$
f_{I P A}=\left(t * \sum_{k \in \delta_{n}} \frac{P_{k, n}\left|\underline{h}_{k} \underline{w}_{k}\right|^{2}}{\sum_{k^{\prime} \in \delta_{n}, k^{\prime} \neq k} P_{k, n}\left|\underline{h}_{k^{\prime}} \underline{w}_{k}\right|^{2}+\eta_{k}^{2}}+B_{I P A}\left(P_{k, n}\right)\right)
$$


Again, by computing the gradient and the Hessian of (16), we can find a solution to the optimization problem in (15) using the Newton with logarithmic barrier method.

Now, the first part of the power allocation problem comes to an end here. The objective of the second part is to reduce the power consumption. Before proceeding further, it is very important to note here that the power allocation of the first part of the algorithm can lead to an infeasible solution. For example, consider the same scenario discussed earlier with the three RREs, and let us focus on the second RRE (the one serving two UEs). Assume that it has two available RBs and that after finishing the first part of the algorithm on the first $\mathrm{RB}$, the UE power allocation was $0.6 \mathrm{P}$, for example and on the second $\mathrm{RB}$, the UE power allocation was $0.5 \mathrm{P}$. Now this RRE should transmit by a total of $1.1 \mathrm{P}$, which is clearly infeasible as it exceeds the constraint per RRE. Consequently, the second part of the IPA algorithm will assure solving the infeasibility problem while reducing the power consumption even further.

Now, with the aim of reducing the power consumption, the second part of the IPA algorithm will scale the power allocation vector resulting from the first part without changing the ratios between its elements so that each element in the vector should never exceed its initial value (the new value should always be less than the initial). For example, considering the same scenario discussed earlier, the initial power allocation vector is: $P A_{n}=\left[\begin{array}{lll}P / 3 & P / 2 & P\end{array}\right]$. If the new power allocation vector is, for example, $\left[\begin{array}{lll}0.5 P & 0.58 P & 0.75 P\end{array}\right]$ then it should be updated so that each element does not exceed its previous value yielding

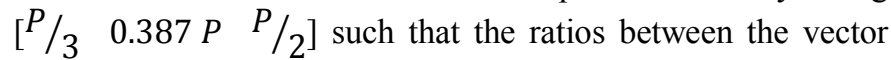
elements are the same. It is clear now that each element does not exceed its previous value leading to a guaranteed feasible solution and the total power consumption is reduced to $1.22 P$ instead of $1.83 P$ in our example.

After finishing the two parts of the algorithm for all the RBs, we still need to make sure that this algorithm enhances the overall throughput and also leads to a reduction in the power consumption. Towards that end, we use the metric defined in [4] and [6], which is the global energy as our stopping criterion. The global energy is the summation of the inverse of the SINR values of all the UEs sharing the same RB. If, after each iteration, the new global energy metric is decreased, then this means that the interference is reduced leading to maximizing the throughput and reducing the power consumption. We then iterate by using the new power allocation vector as the new initial vector (since we are getting better power allocation vector, it does not make sense to stop until reaching the best one) and we can iterate as long as the global energy metric is decreasing.

\section{Performance Evaluation}

In this section, the performance of the proposed algorithms will be investigated. It will be shown that the proposed algorithms significantly outperform the EPA algorithm. We employ the proposed scheduling and precoding based on SLNR in all the simulated algorithms. In our simulation, we consider the urban macrocell channel model detailed in [13]. The UEs are uniformly distributed over the cell coverage area. The frequency is assumed to be $2 \mathrm{GHz}$ and the subcarrier spacing is $15 \mathrm{KHz}$. Each RB has 12 subcarriers; we consider different number of available RBs: 25, 50, 75, and $100 \mathrm{RBs}$, which correspond to the following system bandwidths: 5, 10, 15 , and $20 \mathrm{MHz}$, respectively.

Figs. 1 and 2 show the performance of the proposed joint scheduling algorithm in combination with the OPA, PAR, IPA, and EPA. As shown, PAR significantly increases the overall throughput especially in case of JP-CoMP and IPA significantly increases the overall throughput especially in case of CS-CoMP. In the case of CS-CoMP, the OPA, PAR, and IPA algorithms respectively achieve on average a $77 \%$, $24 \%$, and $37 \%$ throughput gain compared to the EPA as shown in Fig. 1. In the case of JP-CoMP, the OPA, PAR, and IPA algorithms, respectively, achieve on average $85 \%, 71 \%$, and $16 \%$ throughput gains compared to EPA as shown in Fig. 2. It is worth mentioning here, that applying the PAR algorithm in JP-CoMP achieves higher throughput gain than in case of CS-CoMP. This is because the PAR algorithm aims at maximizing the SLNR values for the UEs served by each RRE and since in JP-CoMP, each UE is served by all the RREs, then each RRE optimizes its total transmit power taking all the scheduled UEs into consideration. However, in CS-CoMP, each RRE aims at maximizing the SLNR for only its scheduled UEs.

Fig. 3 shows the normalized average power per RRE. As shown, the RREs applying EPA use their maximum total power. In contrast, the proposed algorithms save a considerable portion of the power consumed while maintaining the overall throughput considerably high as shown in Figs. 1 and 2. The OPA, IPA, and PAR algorithms achieve on average a $26 \%$, $31 \%$, and $21 \%$ power reduction compared to the EPA. It is worth mentioning here, that the IPA algorithm achieves the best performance in terms of energy efficiency, which is expected due to the iteration between its two parts as discussed in Section IV.C.

In Fig. 4, we study the desired accuracy versus Newton iterations. As shown, the figure has a staircase shape where each stair represents an outer iteration and the length of each stair represents the number of inner iterations required for that specific outer iteration. As can be shown, the number of inner iterations decreases with each stair. In other words, the first outer iteration has the maximum inner iterations, while the last outer iteration has the minimum inner iterations. And that is expected because as the number of outer iterations increases, the output of the previous outer iteration becomes a very good starting point and the number of Newton steps needed to compute the next outer iteration becomes small. By means of simulation, it has been found that, $\mu=10$, the outer iteration parameter, is optimum in the sense of number of iterations. In Fig. 4, without loss of generality, we consider $N=100$. 


\section{Conclusions}

We propose scheduling, precoding, and optimized power allocation algorithms aiming at minimizing network power consumption and maximizing the overall data rate in CoMP LTE-A systems. Our results show that the combination of scheduling, precoding, and power allocation can improve network energy efficiency and overall throughput. The proposed algorithms are based on the SLNR metric, which is useful for practical networks due to its reduced computational complexity. Also in this paper, we study the convergence behavior of the three algorithms through observing the number of Newton's iterations for various accuracy values, which is a measure of the computational effort. Simulation results show that the OPA algorithm provides throughput gains of $85 \%$ and $77 \%$ for JP-CoMP and CS-CoMP, respectively, the PAR algorithm provides throughput gains of $71 \%$ and $24 \%$ for JPCoMP and CS-CoMP, respectively, and finally, the IPA algorithm provides throughput gains of $16 \%$ and $37 \%$ for JPCoMP and CS-CoMP, respectively, as compared to EPA.

\section{REFERENCES}

[1] 3GPP R1-050833, "Interference mitigation in evolved UTRA/UTRAN," LGE Electronics.

[2] W. Yu, T. Kwon, and C. Shin, "Joint scheduling and dynamic power spectrum optimization for wireless multicell networks," in CISS, 2010, pp.1-6.

[3] S. Parkvall et al., "LTE-Advanced - evolving LTE towards IMTAdvanced," in VTC 2008-Fall Vehicular Technology Conf. IEEE 68th., 2008, pp. $1-5$.

[4] M. Sawahashi, Y. Kishiyama, A. Morimoto, D. Nishikawa, and M. Tanno, "Coordinated multipoint transmission/reception techniques for LTE-Advanced," IEEE Wireless Communications, vol. 17, no. 3, 2010, pp. 26-34.

[5] X. Hou, E. Bjornson, C. Yang, and M. Bengtsson, "Cell-grouping based distributed beamforming and scheduling for multi-cell cooperative transmission," IEEE International Symposium on Personal, Indoor and Mobile Radio Communications, 2011, pp. 1939-1943.

[6] V. Garcia, C. S. Chen, N. Lebedev, and J.-M. Gorce, "Self-optimized precoding and power control in cellular networks," IEEE International Symposium on Personal, Indoor and Mobile Radio Communications, 2011, pp. 81-85

[7] C. S. Chen and F. Baccelli, "Self-optimization in mobile cellular networks: power control and user association," in Proc. IEEE ICC, 2010, pp. 1-6.

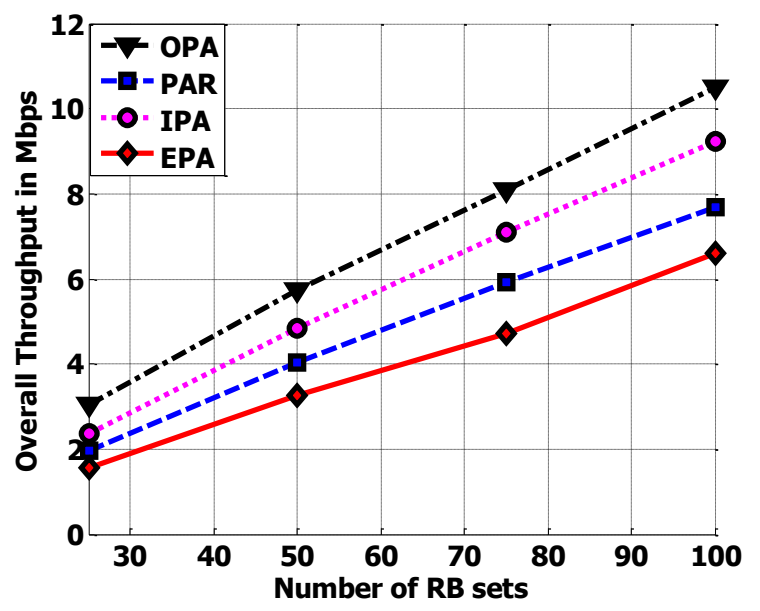

Figure 1. Overall throughput for OPA, PAR, IPA, and EPA assuming CS.
[8] R. A. Abdelaal, M. H. Ismail, and K. Elsayed, "Resource allocation strategies based on the Signal-to-Leakage-plus-Noise Ratio in LTE-A CoMP systems," IEEE WCNC 2012, April 2012.

[9] M. Sadek, A. Tarighat, and A.H. Sayed, "A Leakage-Based Precoding Scheme for Downlink Multi-User MIMO Channels," IEEE Transactions on Wireless Communications, 2007, pp. 1711-1721.

[10] W. Yu, "Multiuser Water-filling in the Presence of Crosstalk," in Proc. IEEE Information Theory Applications Workshop, 2007, pp. 414-420.

[11] G. Song and Y. Li, "Adaptive subcarrier and power allocation in OFDM based on maximizing utility," IEEE VTC, 2003, pp. 905-909.

[12] S. Boyd and L. Vandenberghe, "Convex Optimization,"Cambridge University Press, 2004. www.stanford.edu/ boyd.

[13] IST-WINNER II, D1.1.2 , "WINNER II Channel Models,"2007, http://www.ist-winner.org/deliverables.html.

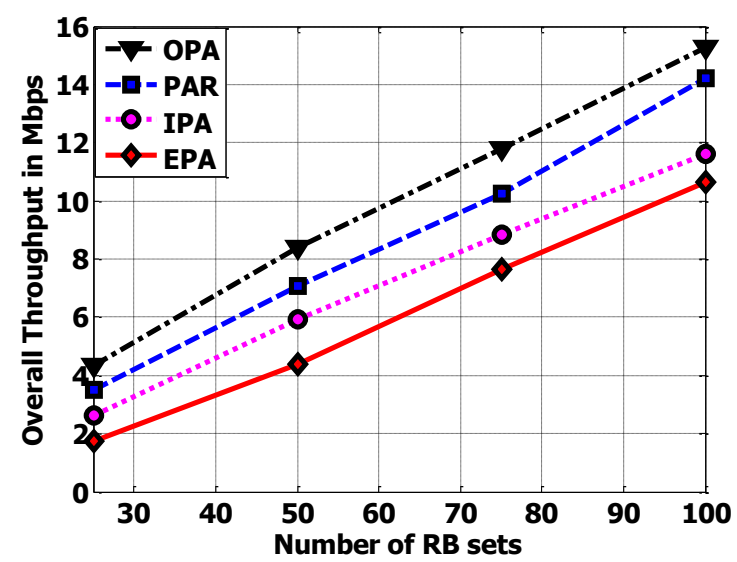

Figure 2. Overall throughput for OPA, PAR, IPA, and EPA assuming JP.

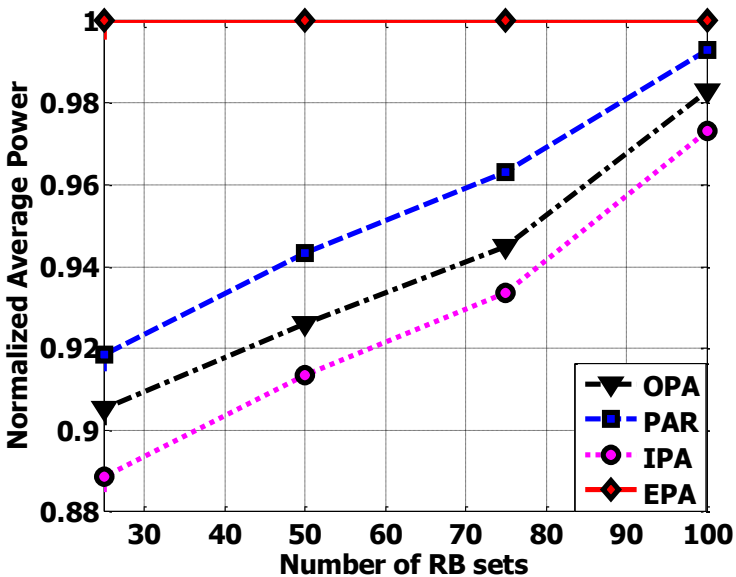

Figure 3. Normalized average power for OPA, PAR, IPA, and EPA.

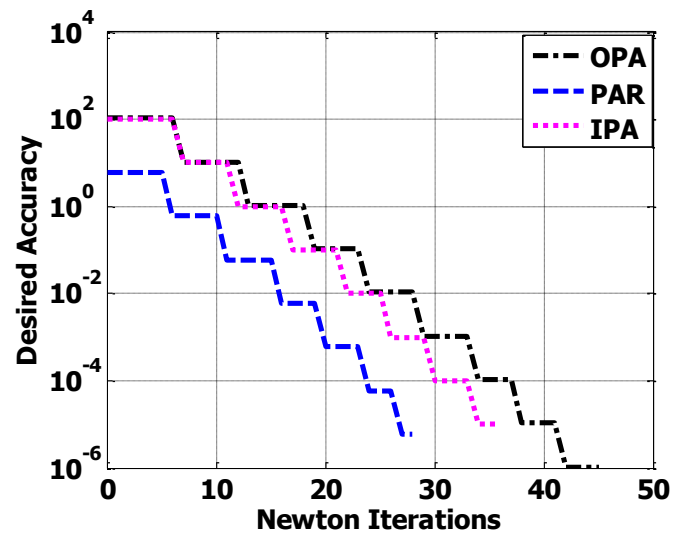

Figure 4. Desired accuracy versus Newton iterations for OPA, PAR, and IPA. 